\title{
Model of pp and AA collisions for the description of long-range correlations
}

\author{
Vladimir Kovalenko \\ Saint Petersburg State University \\ E-mail: nvkinferambler.ru \\ Vladimir Vechernin* \\ Saint Petersburg State University \\ E-mail: vechernin@gmail.com
}

Soft processes in pp and AA interactions are considered in the framework of phenomenological model with color strings formation and fusion. Elementary parton collisions are realized in the model as the interaction of two colour dipoles from projectile and target nucleons. Modeling of the exclusive distributions of parton momentum fractions and transverse coordinates is performed. The interaction of colour strings in transverse plane is carried out in the framework of local string fusion model with the introduction of the lattice in the impact parameter plane and taking into account the finite rapidity length of strings. The parameters were fixed with experimental data on pp total inelastic cross section and charged multiplicity.

The model was used for the calculation of long-range correlations between the multiplicities (n) and the mean transverse momenta $(\mathrm{pt})$ of charged particles. The dependence of $\mathrm{n}-\mathrm{n}, \mathrm{pt}-\mathrm{n}, \mathrm{pt}-\mathrm{pt}$ correlations on the width and position of the backward and forward rapidity windows was studied. Note that the model enables to describe the AA interactions without referring to the Glauber picture based on the concept of elementary nucleon-nucleon collisions. In this connection the charged multiplicity, the mean numbers of participant nucleons and binary collisions and their variances in the case of $\mathrm{PbPb}$ collisions were calculated and compared with the predictions of alternative models and the experimental data. The influence of different ways of centrality determination on the multiplicity fluctuations and long-range correlations was also discussed.

XXI International Baldin Seminar on High Energy Physics Problems September 10-15, 2012

JINR, Dubna, Russia

${ }^{*}$ Speaker. 


\section{Introduction}

The present work is devoted to development of Monte Carlo model for the description of soft proton-proton and heavy ion collisions at high energy. Due to inapplicability of usual QCD methods in this region, such studies are semi-phenomenological. One of the models, which successfully describe particle production in soft region, is so-called quark-gluon string model [1,2]. The model involves two-stage scenario of particles production: at the first stage extended in rapidity objects (quark-gluon strings, or colour tubes) are stretched between the partons of colliding hadrons, and they fragment into observable particles at the second stage.

In order to study these extended in rapidity strings it was proposed to measure long-range correlations between observables from two rapidity windows, separated by a gap.

As these objects are finite in transverse plane, the overlapping of them and possible interactions can lead to non-trivial effects. Such interaction of the strings is considered in so-called string fusion model [3-5]. In particular, this model predicts the existence of non-zero long range correlations between multiplicity and mean transverse momentum (pt-n) and pt-pt correlations. The theoretical study of long-range correlations in the framework of string fusion model is carried out using Monte Carlo simulations [6-10]. The goal of this work is to develop detailed Monte Carlo model, taking into account finite rapidity width of the strings and their fusion at the transverse plane both for pp and AA collisions.

\section{The model}

In order to provide parton-string model, which is suitable for reliable description of fluctuations and correlations, one should take into account not only inclusive, but also exclusive parton distributions as well as the details of the string formation and fusion.

\subsection{Partonic picture of p-p collision}

The form of inclusive distributions on the momentum fraction for $\mathrm{N}=2 \mathrm{n}$ partons is taken from $[11,12]$ :

$$
\begin{aligned}
f_{u}(x) & =f_{\bar{u}}(x)=C_{u, n} x^{-\frac{1}{2}}(1-x)^{\frac{1}{2}+n}, \\
f_{d}(x) & =f_{\bar{d}}(x)=C_{d, n} x^{-\frac{1}{2}}(1-x)^{\frac{3}{2}+n}, \\
f_{u d}(x) & =C_{u d, n} x^{\frac{3}{2}}(1-x)^{-\frac{3}{2}+n}, \\
f_{u u}(x) & =C_{u u, n} x^{\frac{5}{2}}(1-x)^{-\frac{3}{2}+n} .
\end{aligned}
$$

At $n>1$ the sea quarks and antiquarks have the same distribution as the valence quarks. Poisson distribution for the number of quark-antiquark (diquark) pairs $(n)$ is assumed with some parameter $\lambda$, omiting the case of $n=0$,

For corresponding exclusive distribution for an arbitrary number of quark-antiquark pairs we find:

$$
\rho\left(x_{1}, \ldots x_{N}\right)=c \cdot \prod_{j=1}^{N-1} x_{j}^{-\frac{1}{2}} \cdot x_{N}^{\alpha_{N}} \delta\left(\sum_{i=1}^{N} x_{i}-1\right)
$$


The valence quark is labelled by $\mathrm{N}-1$, the diquark - by $\mathrm{N}$, and the other refers to sea quarks and antiquarks. $\alpha_{N}=3 / 2$ (ud-diquark), $\alpha_{N}=5 / 2$ (uu-diquark).

Corresponding algorithms for modelling of such distributions are discussed in [10].

The exclusive distribution in the impact parameter plane is constructed on the following suppositions:

1. The position of the mass centre is fixed: $\sum_{j=1}^{N} \vec{r}_{j} \cdot x_{j}=0$.

2. The inclusive distribution of each parton is the 2-dimensional Gaussian distribution.

3. The normalization condition is $\left.\left\langle r^{2}\right\rangle=<\frac{1}{N} \sum_{j=1}^{N} r_{j}^{2}\right\rangle=r_{0}^{2}$,

where the parameter $r_{0}^{2}$ is connected with the mean square radius of the proton: $\left.<r_{N}^{2}\right\rangle=\frac{3}{2} r_{0}^{2}$.

For the description of inelastic proton-proton collisions we assume that the elementary parton collision is implemented as an interaction of two color dipoles consisting of a valence quark and diquark, or of a quark-antiquark pair. The probability amplitude of interaction of two dipoles with coordinates $\left(\vec{r}_{1} \vec{r}_{2}\right)$ and $\left(\vec{r}_{3} \vec{r}_{4}\right)$ in the impact parameter plane is given by [13]:

$$
f=\frac{\alpha_{S}^{2}}{8} \ln ^{2} \frac{\left(\vec{r}_{1}-\vec{r}_{3}\right)^{2}\left(\vec{r}_{2}-\vec{r}_{4}\right)^{2}}{\left(\vec{r}_{1}-\vec{r}_{4}\right)^{2}\left(\vec{r}_{2}-\vec{r}_{3}\right)^{2}}
$$

where $a_{S}-$ is a constant.

The value of $\alpha_{S}$ is assumed to be an effective coupling constant, and $\alpha_{S}$ is used as a fitting parameter for better description of the experimental data, it is assumed that the value does not depend neither on the energy, nor on the number of quark-antiquark pairs in the proton.

Taking into account the confinement effects $[13,14]$ leads:

$$
f=\frac{\alpha_{s}^{2}}{2}\left[K_{0}\left(\frac{\left|\vec{r}_{1}-\vec{r}_{3}\right|}{r_{\max }}\right)+K_{0}\left(\frac{\left|\vec{r}_{2}-\vec{r}_{4}\right|}{r_{\max }}\right)-K_{0}\left(\frac{\left|\vec{r}_{1}-\vec{r}_{4}\right|}{r_{\max }}\right)-K_{0}\left(\frac{\left|\vec{r}_{2}-\vec{r}_{3}\right|}{r_{\max }}\right)\right]^{2}
$$

which provides an exponential decrease of collision probability of two protons with large impact parameter.

In the eikonal approximation $[14,15]$ the probability of interaction of two dipoles is given by:

$$
p_{i j}=1-e^{-f_{i j}} .
$$

The total probability of inelastic interaction of two ptorons is $p=1-e^{-\sum_{i, j} f_{i j}}$, where the summation is made over all the dipoles.

For more detiled discussion please refer also to $[9,10]$.

\subsection{Calculation of observables}

Probabilities of dipole interaction, obtained in the previous section, are used for the construction of the collision matrix. It should be noted, that a dipole can interact only with one other dipole, so filling of the interaction matrix starts from the valence dipole.

The next step is the generation of strings in rapidity space. Rapidity ends of a string $y_{\min }, y_{\max }$ are determined from the kinematic condition of a string decay only on two particles with an average 
$p_{t}=0.3 \mathrm{GeV}$ and masses $m_{\pi}=0.15 \mathrm{GeV}$ (for pion - in case of quarl-antiquatk string) or $m_{p}=$ $0.94 \mathrm{GeV}$ (for nucleon - in case of diquark at the end of the string).

The strings, that are too short, are excluded from consideration by the requirement that the sum of the masses of the particles produced should be less than the mass of the string, ie $\sqrt{s} x_{A} x_{B}$, where $x_{A}, x_{B}$ - momentum fractions of the partons at the ends of the string. Transverse coordinates of the center of the strings are equal to the arithmetic mean of the corresponding coordinates of the partons at the ends.

Due to finite transverse size of the strings they overlap in the impact parameter plane. The interaction of colour strings in transverse plane is carried out in the framework of local string fusion model [5] with the introduction of the lattice in the impact parameter plane. [6, 7, 16-18]. Cellular variant of string fusion has been chosen in order to simplify taking into account finite rapidity length of strings, because, as it had been shown, numerical results differ little in several string fusion model variants $[6,8]$.

According to this model, mean multiplicity of charged particles and mean $p_{t}$ originated from the area $S_{k}$, where k strings are overlapping are the following:

$$
\langle\mu\rangle_{k}=\mu_{0} \sqrt{k} \frac{S_{k}}{\sigma_{0}} \quad\left\langle p_{t}\right\rangle_{k}=p_{0} \sqrt[4]{k}
$$

where $\sigma_{0}=\pi r_{s t r}^{2}-$ string transverse area

In the discrete model transverse plane is considered as a grid with the cell area equals to string transverse area and strings are fused if their centers occupy the same cell.

Finite rapidity width of strings is also taken into account[10]: rapidity bins are defined by strings edges, so every rapidity interval contains constant number of strings, then bins are processed separately with the final summation over whole rapidity axis.

The Monte Carlo model was implemented as C++ class, and in order to get results with high statistics, distributed grid computing have been involved [19].

\section{Parameters fixation}

In the framework of our model the growth of multiplicity and total inelastic cross section is achieved by increasing the number of quark-antiquark pairs (parameter $\lambda$ ). The value of $\lambda$ is turned from the experimental data on the total inelastic cross section [20].

The simulations are conducted in two phases. The first step is devoted to determination of $\sigma_{p p}^{i n}$ for the given $\lambda$. Using experimental data, the dependence of $\lambda$ on collision energy is obtained (example is shown at fig. 1).

One should note the importance of sea quark presence even at low energy [9]. 


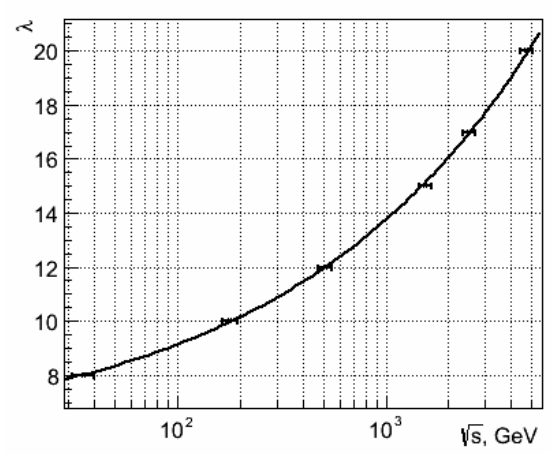

Figure 1: Connection of parameter $\lambda$ and the energy for the case of $\alpha_{s}=0.7$.

At the second step all other calculations are carried out for the given energy.

The value of mean multiplicity per rapidity from one single string $\mu_{0}$ is fixed at one point at low energy.

The other parameters of the model were chosen as follows: for $r_{\max } \simeq 0.2-0.3 \mathrm{fm}$; ratio $r_{\max } / r_{0}$ is chosen to be 0.5 ; constant $\alpha_{S}$ is rixed for best description of the dependence of charged multiplicity on energy [21]. For the string radius $r_{s t r}$ we considered several possible values: $0.2 \mathrm{fm}, 0.3 \mathrm{fm}, 0.4 \mathrm{fm}$, which are within the estimates [22, 23, 16, 17], used for the description of long-range correlations in nucleus-nucleus collisions.

The results of parameters fixing are presented in Table 1, the corresponding plot for the multiplicity shown in Fig. 2.

Table 1:

\begin{tabular}{|c|c|c|}
\hline$r_{s t r}=0.2 \mathrm{fm}$ & $\alpha_{S}=0.7$ & $\mu_{0}=0.92$ \\
\hline$r_{s t r}=0.3 \mathrm{fm}$ & $\alpha_{S}=0.5$ & $\mu_{0}=1.02$ \\
\hline$r_{s t r}=0.4 \mathrm{fm}$ & $\alpha_{S}=0.5$ & $\mu_{0}=1.12$ \\
\hline
\end{tabular}




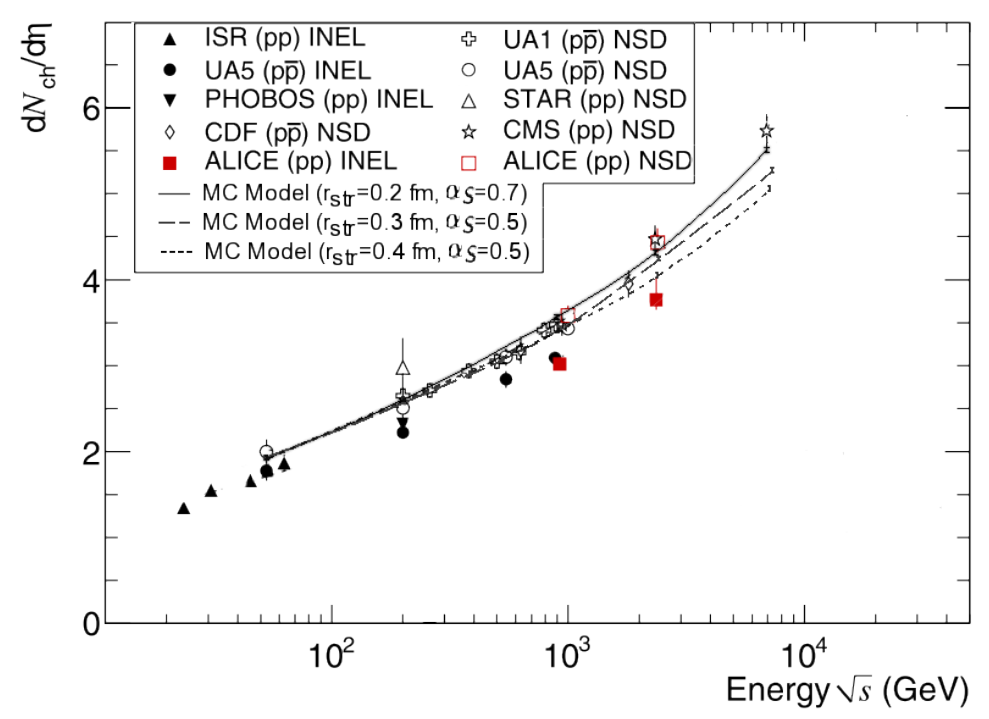

Figure 2: Chaged-particle multiplicity in p-p collisions. Calculations of the Monte Carlo model and experimental data $[21,24]$.

The calculation results show good description of the multiplicity in a wide energy range, and taking into account the results of the LHC at $7 \mathrm{TeV}$ we use the first set of parameters $\left(r_{s t r}=0.2 \mathrm{fm}\right.$, $\left.\alpha_{S}=0.7, \mu_{0}=0.92\right)$ for further studies.

\section{Calculation of correlation functions}

The correlation function between two observable B (backward) and F (forward) from different rapidity windows is defined as the mean value of $\mathrm{B}$ with fixed $\mathrm{F}[6]$ :

$$
f_{B-F}(F)=\langle B\rangle_{F} .
$$

The correlation coefficient represents the slope of correlation function:

$$
b_{B-F}=\left.\frac{d f(F)}{d F}\right|_{F=<F>} .
$$

Often it is useful to switch to normalized variables: $B \rightarrow B /\langle B\rangle, F \rightarrow F /\langle F\rangle$, in this case $p_{t}-n$ correlation coefficient would become dimensionless, and both $n-n$ and $p_{t}-p_{t}$ correlation coefficients would not change in case of symmetrical windows. Thus we use the following definitions:

$$
\begin{aligned}
b_{n n}= & \left.\frac{<n_{F}>}{<n_{B}>} \cdot \frac{d<n_{B}>}{d n_{F}}\right|_{n_{F}=<n_{F}>}, \\
b_{p_{t}-n}= & \left.\frac{<n_{F}>}{<p_{t B}>} \cdot \frac{d<p_{t B}>}{d n_{F}}\right|_{n_{F}=<n_{F}>}, \\
b_{p_{t}-p_{t}}= & \left.\frac{<p_{t_{F}}>}{<p_{t B}>} \cdot \frac{d<p_{t_{B}}>}{d p_{t F}}\right|_{p_{t F}=<p_{t F}>} .
\end{aligned}
$$

In practice, the experimental determination of the correlation coefficient and Monte Carlo simulation is performed by obtaining fitting of $f(F)$ by linear function from $\langle F\rangle-\sigma_{\langle F\rangle}$ to $\langle F\rangle+$ $\sigma_{\langle F\rangle}$, where $\sigma_{\langle F\rangle}=\sqrt{\left\langle F^{2}\right\rangle-\langle F\rangle^{2}}$. This method is used in this paper. 
For the calculation of these correlations in the framework of string fusion model the occupancy $\eta_{i}$ of each cell is defined from string configuration and one assumes, that every cell of the lattice emit particles according to Poisson distribution with parameter equal to $\eta_{i} \cdot \mu_{0} \Delta y$. The mean event transverse momentum is generated with Gaussian distribution with mean [7] $\left(\sum_{i} n_{i} \alpha_{i}\right) /\left(\sum_{i} n_{i}\right)$ and variance $\sum_{i} n_{i} D_{i}$, where $\alpha_{i}=\sqrt[4]{p_{0}}, D_{i}=\sqrt{\eta_{i}} D, D=\gamma p_{0}$.

The coefficient $\gamma$ is the coefficient of proportionality between the average transverse momentum and root of the variance of transverse momentum from one single string. This parameter is assumed to be independent of the energy. As in [7], we used the value $\gamma=0.61$.

Obtained by this way mean event transverse momentum and multiplicity in both forward and backward windows are used to will $2 \mathrm{~d}$ histogram (fig. 14) and correlation function is obtained by regression.

This way in case of $n-n$ and pt-n correlations is fully equivalent to the one, described in $[6,10]$ and also coincides with the one, used for pt-pt correlations [7].

\section{Results on pp interaction}

\section{Correlation functions}

Correlation functions for p-p collisions are sown at fig. 3, 4 and 5. One should note the nonlinearity of all types correlation functions with significant deviation from linear function occuring only outside the region $\left\langle n_{F}\right\rangle-\sigma_{F}<n_{F}<\left\langle n_{F}\right\rangle+\sigma_{F}$, which is used for determination of correlation coefficients.

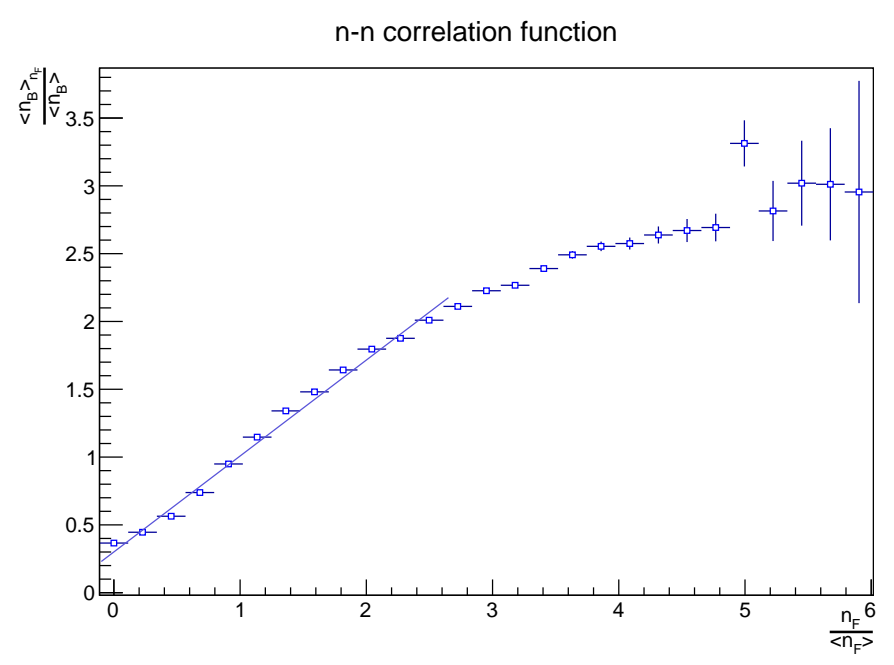

Figure 3: $\mathrm{n}-\mathrm{n}$ correlation function for pp collisions at $7 \mathrm{TeV}$, calculated in MC model. Rapidity windows are $(-0.8,0)$ and $(0,0.8)$. 


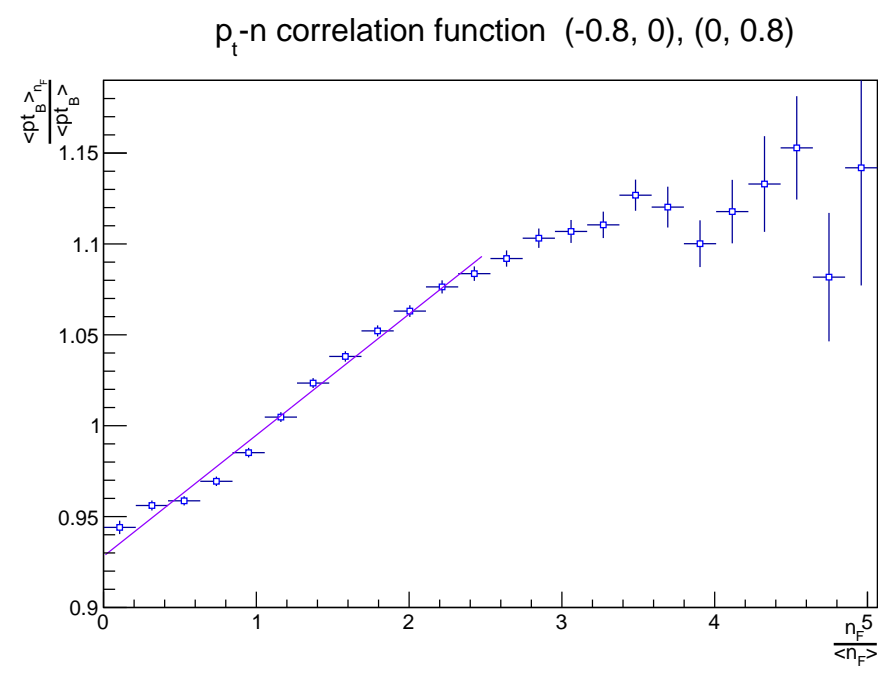

Figure 4: pt-n correlation functions for pp collisions at $7 \mathrm{TeV}$, calculated in MC model.

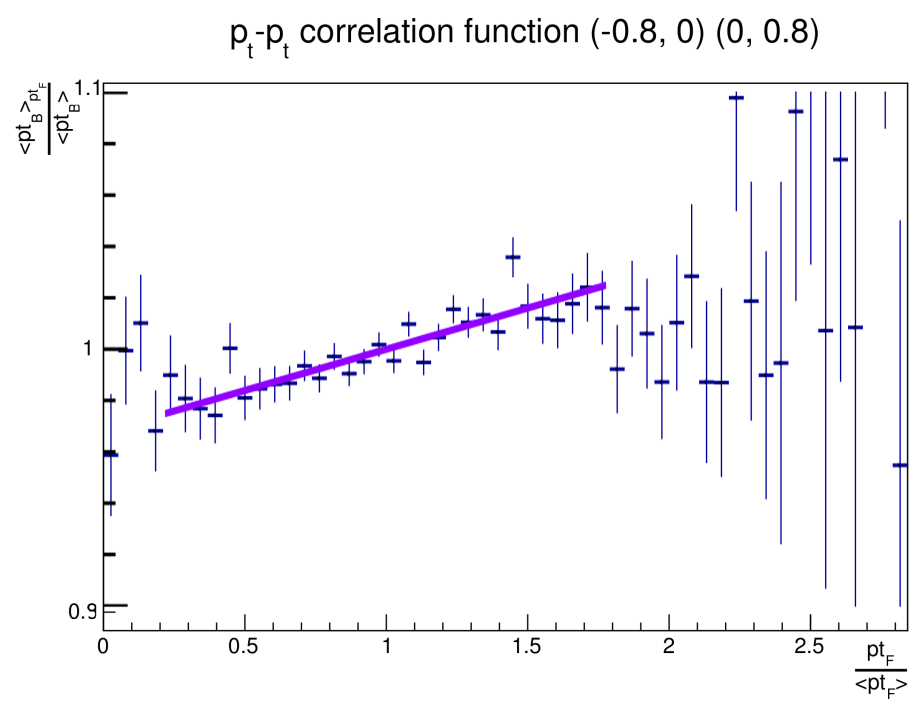

Figure 5: pt-pt correlation functions for pp collisions at $7 \mathrm{TeV}$, calculated in MC model.

\section{Correlation coefficients}

We also studied the dependence of correlation coefficients on the width of rapidity windows.

Firstly we consider the dependence of correlation coefficients on the width of backward rapidity window with fixed forward window at $(0.6,0.8)$. The configurations of the windows used are shown at the fig. 6. Correlation coefficients are calculated for pp collisions at $7 \mathrm{TeV}$ and results are shown at the fig. 7 . 


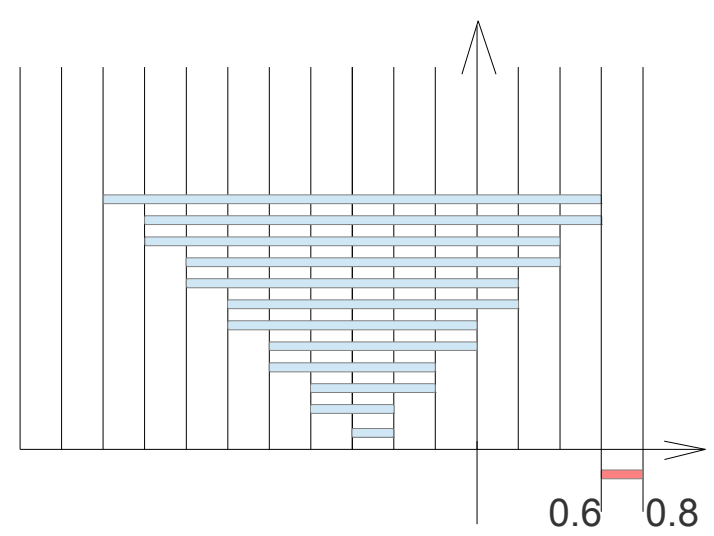

Figure 6: Configuration of rapidity windows with fixed forward window.
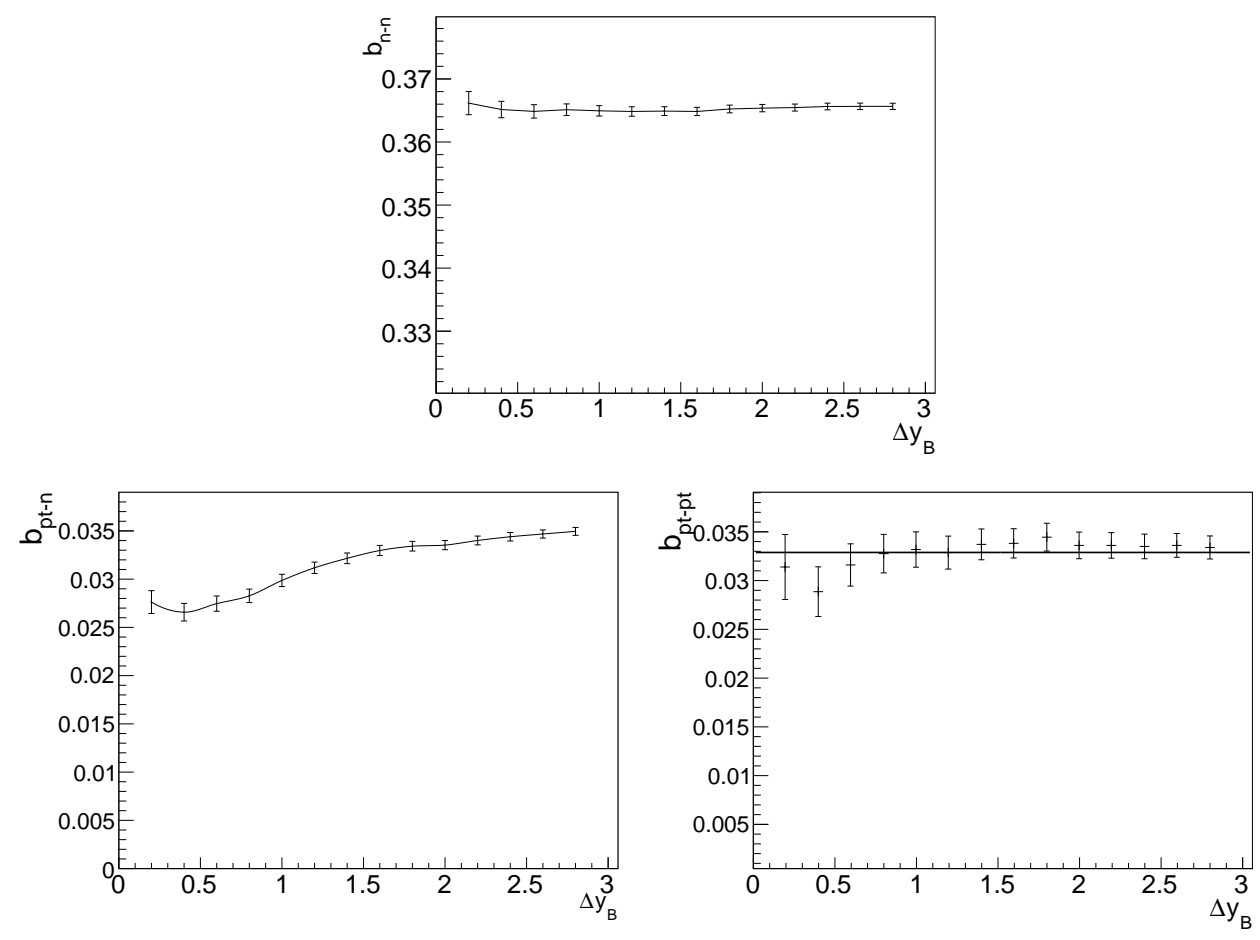

Figure 7: n-n, pt-n and pt-pt correlation coefficients in p-p collisions at $7 \mathrm{TeV}$ as a function of the width of backward window.

The dependence of correlation coefficients on the width of forward rapidity windows is shown at fig. 9 . 


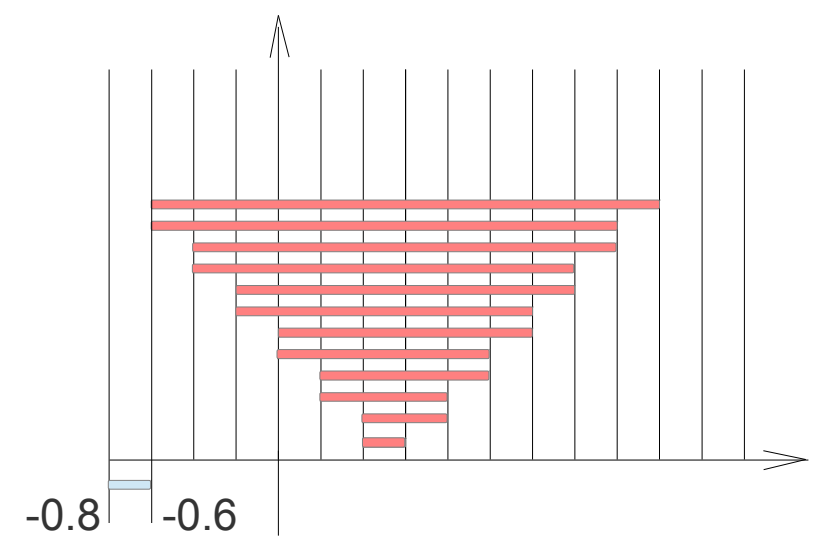

Figure 8: Configurations of rapidity windows with fixed backward window.
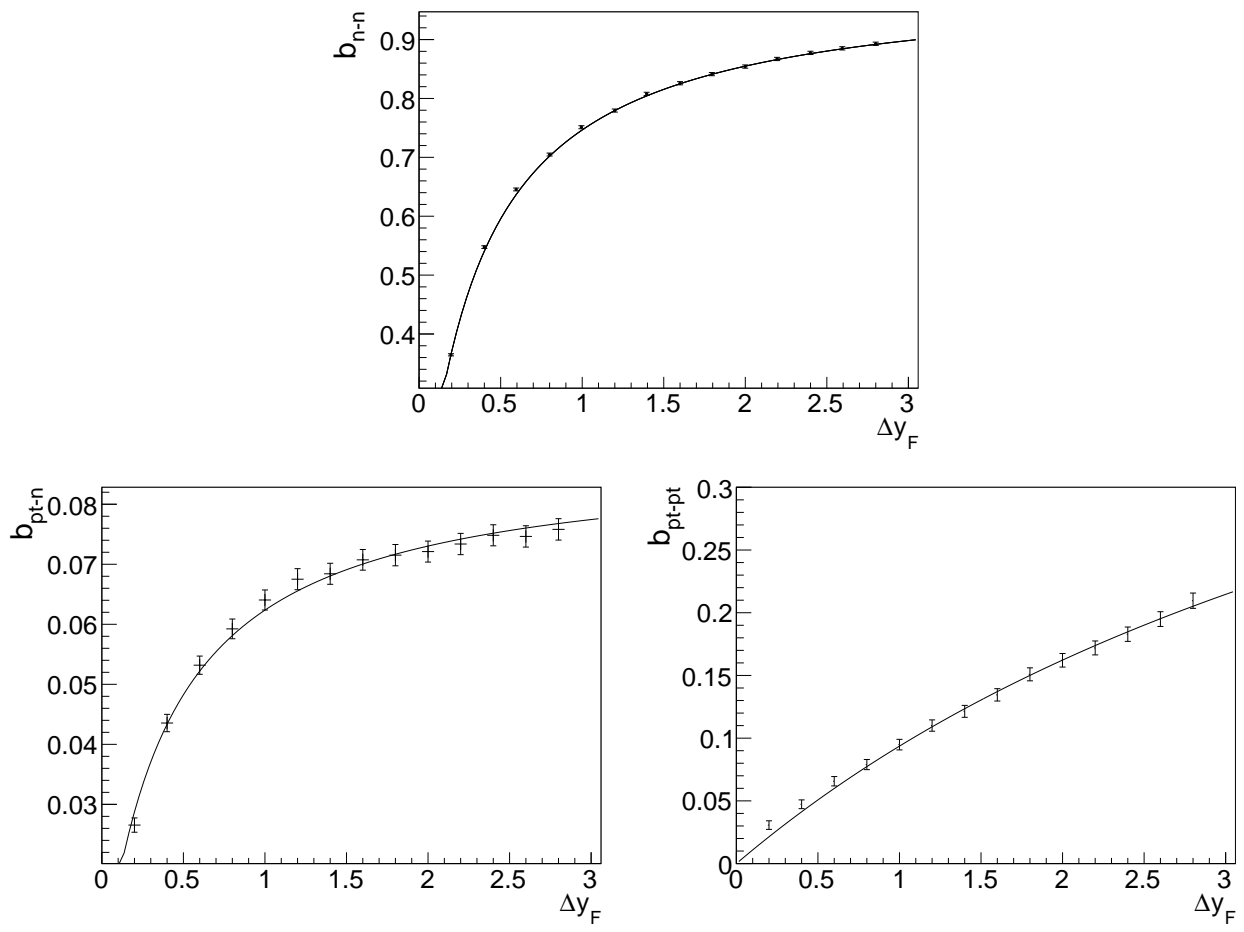

Figure 9: n-n, pt-n and pt-pt correlation coefficients in p-p collisions at $7 \mathrm{TeV}$ as a function of the width of forward window.

The values calculated in the Monte Carlo program are fitted by the formula

$$
b=\beta \frac{\Delta y_{F}}{\Delta y_{F}+k} .
$$

Fit parameters, obtained for n-n, pt-n and pt-pt correlations are shown in the table 2. 
Table 2: Parameters of a fitirovaniye of coefficients of correlation

\begin{tabular}{|c|c|c|}
\hline Type of correlation & $\beta$ & $k$ \\
\hline$n-n$ & $1.000 \pm 0.002$ & $0.34 \pm 0.01$ \\
\hline$p_{t}-n$ & $0.088 \pm 0.002$ & $0.41 \pm 0.02$ \\
\hline$p_{t}-p_{t}$ & $0.53 \pm 0.05$ & $4.6 \pm 0.6$ \\
\hline
\end{tabular}

\section{Discussion of results}

The absence of dependence of $n-n$ correlation coefficient on the width of a backward window corresponds to the predictions of the models of independent emitters [25] and to asimptotical expressions, obtained in the string fusion model $[6,7,26]$ in the limit case of high string density.

Small dependence of $p_{t}-n$ correlation coefficient on the width of a forward window can be caused by dependence of this coefficient on the gap between windows (which is stronger for $p_{t}-n$ correlation, than for $n-n$ ). An additional source of such dependence may be constraint $n_{B}>0$. In fact, if we select an event with non-zero number of particles in backward window, we potentially select higher-multiplicity event, probably, with higher mean transverse momentum and this can change the value of the correlation coefficient in the relative variables. In wide backward window this constraint becomes negligible.

$P_{t}-p_{t}$ correlations also do not depend on width of a back window within error bars.

The dependence of a coefficient of correlation on the width of a forward window is completely described by the formula (3.1); for $n-n$ correlation $\beta=1$ is found, that is in full agreement with the predictions of the model of the independent emitters [25]. The fact, that the value $k$ for $n-n$ and $p_{t}-n$ correlations does not coincide, as it was predicted for high string density [6], means that the density of strings, where the asymptotic formula is applicable, is not reached in p-p collisions at $7 \mathrm{TeV}$.

One should notice that $p_{t}-p_{t}$ correlations strongly depend on width of forward rapidity window, and at wide sufficiently $\Delta y_{F}$ dominate over $p_{t}-n$ correlations.

From the physical point of view that fact that correlation coefficients depend only on the width of a forward window means $[16,23]$ that the dynamic variable in the forward window is the one that classifies events, and the wider window is, the closer this classification coincides with the one by string configurations, and hence, the more this value is correlated with a variable in another rapidity window.

In addition, one should notice that obtained dependence of correlation coefficients on the width of rapidity windows can be confirmed in experiment; this is done for $n-n$ correlations [27, 28], and used for the efficiency corrections. 


\section{Dependence of correlation coefficients on the gap between rapidity windows}

The dependence of correlation coefficients on the provision of rapidity windows was also studied in the model. The symmetric windows of the width 0.8 rapidity units were chosen. The results are shown at the fig. 10.
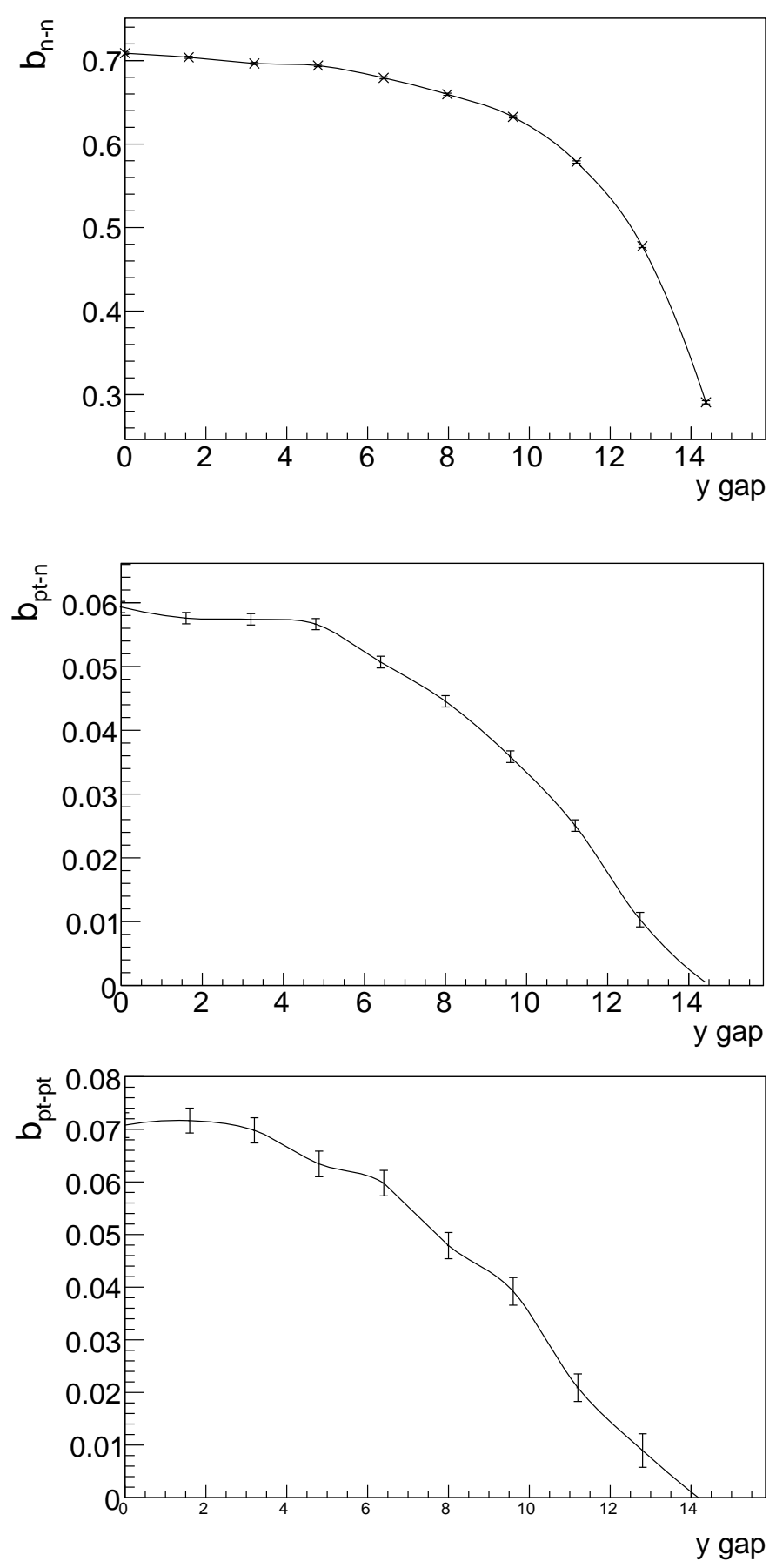

Figure 10: Dependence of n-n, pt-n and pt-pt correlation coefficients on the gap between rapidity windows 
All three types of correlations show similar behavior and decrease at high gap in the same way, as rapidity distribution of charged particles.

It is noticeable that both $p_{t}-n$ and $p_{t}-p_{t}$ correlation coefficients reach zero at wide enough rapidity gap. It can be it is explained as follows: only the lengthiest valence strings contribute to these windows far away from midrapidity, therefore there is no strings to fuse in this area, and consequently there are no $p_{t}-n$ and $p_{t}-p_{t}$ correlation. Multiplicity-multiplicity correlations remain due to fluctuation of the numbers of strings (volume fluctuation): zero and one.

\section{Nucleus-nucleus collisions}

For the description of nucleus-nucleus collisions at high energy the Glauber model is is widely used [29, 30], which is based on an assumption of incoherent superposition of single nucleonnucleon collisions. It is supposed that trajectories of nucleons in a nuclei are approximately straight lines and all consequent collisions are occurred at the same cross section and with identical mean number of produced charged particles (the same as for pp collision).

Thus, multiplicities in pp and AA collisions are connected by the following:

$$
N_{c h}^{A A}=N_{c o l} N_{c h}^{p p}
$$

In such manner Glauber model considerably overestimate charged multiplicity in comparison with experimental data and contains obvious breaking of the energy conservation: indeed, the in such picture same part of energy can go to the particle production several times [31].

In order to get an agreement with experimental data one should use instead of (4.1) some interpolations:

$$
N_{c h}=C\left(x N_{\text {part }}+(1-x) N_{\text {coll }}\right) .
$$

With turned parameters $C$ and $x$ one can achieve of distribution of charged multiplicity consistent with experimental data. The Glauber model is used in such manner for the definition of number of participating nucleons in those experiments where it is impossible to determine it directly [32].

This problem is considered also in modified Glauber model [31], where energy loss is taken into account effectively. There are also several models [33-35] representing "The Glauber on a parton level".

In the present paper we develop a Monte Carlo model without referring to the Glauber picture based on the concept of elementary sequential nucleon-nucleon or partonic collisions. We implement direct generalization of pp model, described above.

The initial arrangement of nucleons is done with standard Woods-Saxon distribution: $\rho_{A}(r)=\frac{\rho_{0}}{1+\exp [(r-R) / d]}$ with $R=1.07 \cdot A^{1 / 3} \mathrm{fm}, d=0.545 \mathrm{fm}$.

Nucleons are treated as set of the dipoles; the elementary collisions it is carried out by means of formulas (2.3 - 2.4). The nucleon is considered as participant if at least one of its dipoles faced a dipole of other nucleus. There is no additional parameter turning. The set of observables is calculated in the same way as for proton-proton collisions. 

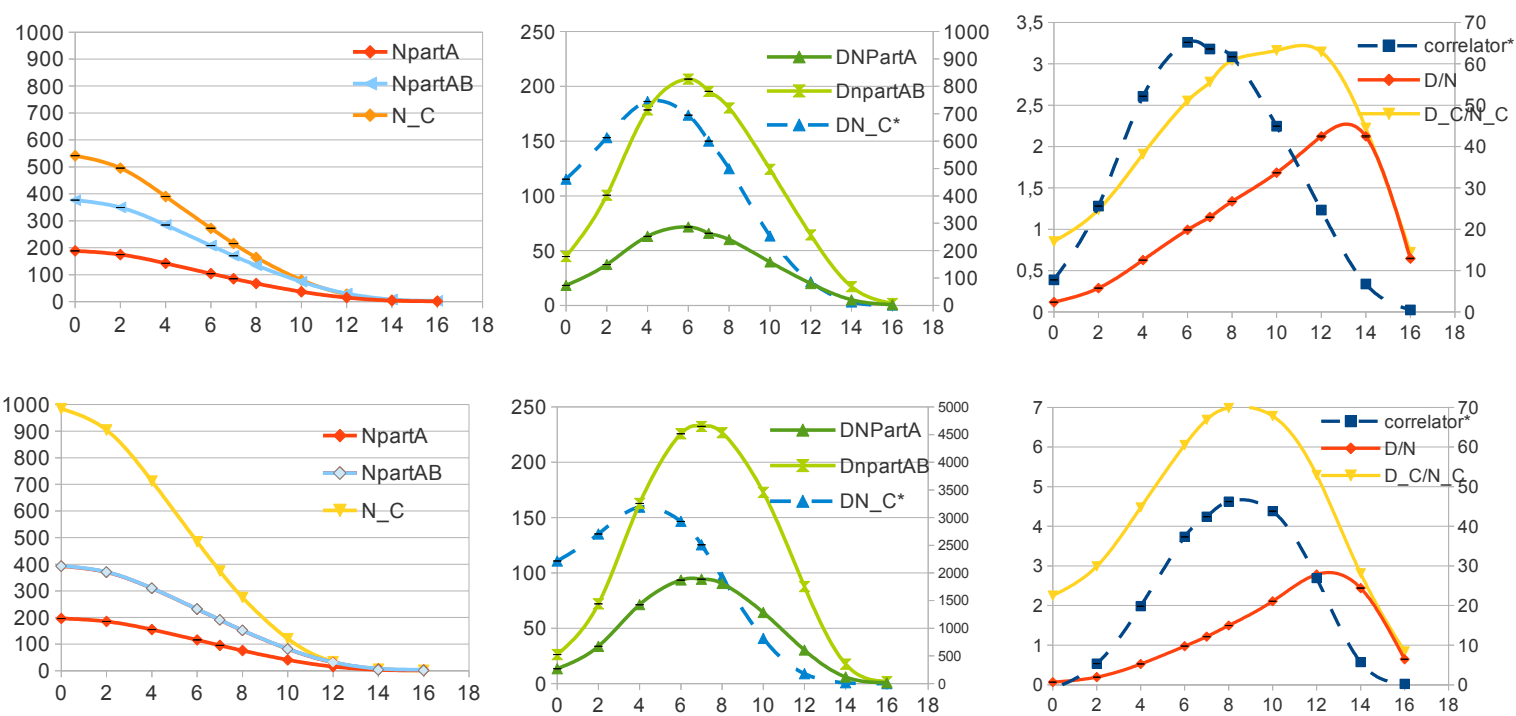

Figure 11: Number of participant nucleons, number of binary collisions (left), their variances (middle), scaled variances and correlator $2\left(\left\langle N_{\text {part }_{A}} N_{\text {part }_{B}}\right\rangle-\left\langle N_{\text {part }_{A}}\right\rangle\left\langle N_{\text {part }_{B}}\right\rangle\right)$ (right): our Monte-Carlo model without Glauber picture (up), and calculations in the Glauber model at $\sigma_{N N}=34 \mathrm{mb}$ (down).

note: additional scale (at the right) is used for variables, marked by star.

In the framework of the $\mathrm{MC}$ model we calculated the number of participants, binary collisions and their variances for $\mathrm{PbPb}$ collisions at low energy $\left(\sigma_{N N}=34 \mathrm{mb}\right)$ at fixed impact parameter and compare them with results of Glauber model calculations (fig. 11).

The results show practically the complete coincidence of predictions of two models on the number of participated nucleons, while the number of binary collisions is almost twice less. There is qualitatively similar behavior of variances of number of participants and number of collisions in these two models; quantitatively in the Glauber model fluctuations appear higher.

The calculations carried out by the author in the Glauber model are close with analytical and numerical calculations $[36,37] \sigma_{N N}=31.4 \mathrm{mb}$.

The decrease of the number of binary collisions can be explained by the following: in every nucleus-nucleus collision each dipole can interact with other one only once, and the energy of colliding partons directly goes to particle production. It reduces additional probability of further interactions of the nucleons with already "used" partons. Thus, such picture respects the energy conservation.

The charged multiplicity per rapidity over a half of number of participating nucleons at the LHC energy is shown at the fig. 12 and compared with ALICE experimental data.

In general, we achieved a good agreement, remaining discrepancy probably could be reduced by additional parameters turning, taking into account some results on $\mathrm{p}-\mathrm{A}$ or AA scattering. 


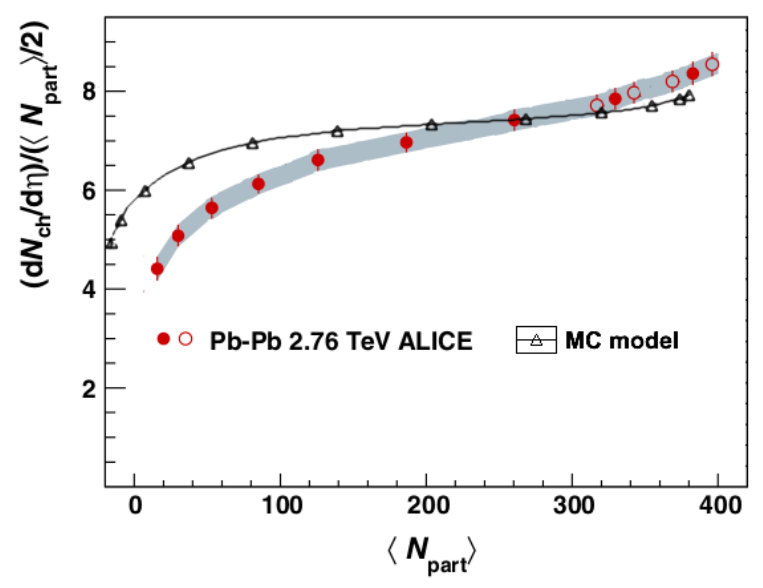

Figure 12: Dependence of $\left(d N_{c h} / d \eta\right) /\left(N_{\text {part }} / 2\right)$ on the number of participants for $\mathrm{Pb}-\mathrm{Pb}$ collisions at $\sqrt{s_{N N}}=2.76 \mathrm{TeV}$. Results of MC calculations and experimental data [32].

On fig. 13 long-range correlation coefficients of $n-n, p_{t}-n$ and $p_{t}-p_{t}$ correlations are shown for $\mathrm{PbPb}$ collisions at the energy of LHC $(2.76 \mathrm{TeV})$. Two different configurations of forward and backward rapidity windows were considered: windows in width 0.8 and 2 rapidity units.

It is noticeable, that even at the fixed impact parameter $n-n$ correlation are very strong. The correlation coefficient decreases in the central collisions that it expected due to string fusion effects (decrease of $\mathrm{n}-\mathrm{n}$ correlation) are important in central $\mathrm{PbPb}$ collisions. High value of $\mathrm{n}-\mathrm{n}$ correlations in semi-central and peripheral collisions are due to volume fluctuation effects.

The large value of $p_{t}-p_{t}$ correlations is remarkable and dominates over $p_{t}-n$ correlations. This fact is in agreement with predictions [7] stated that at LHC energies $p_{t}-p_{t}$ correlation coefficient can reach the level of $n-n$ correlations. 

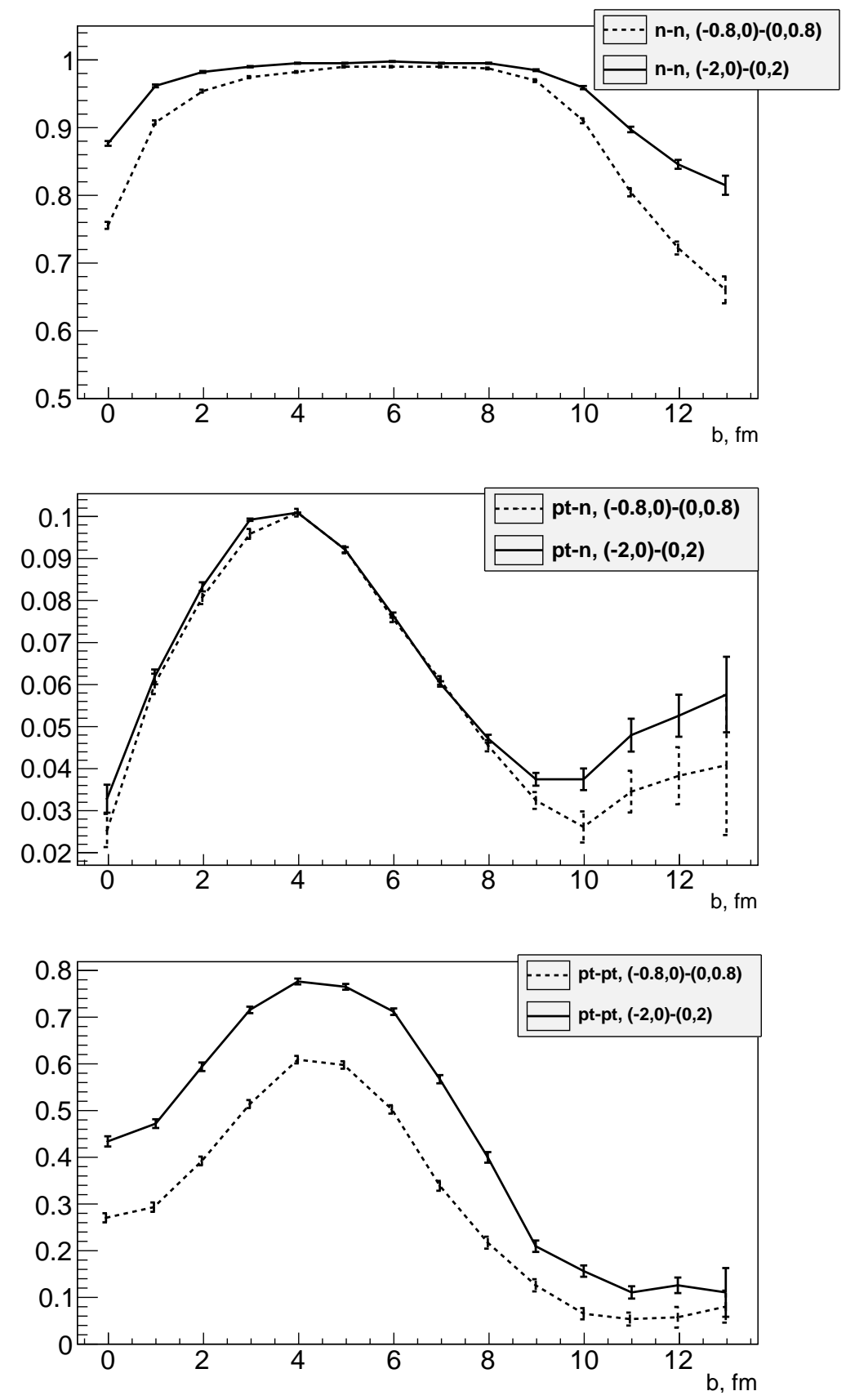

Figure 13: N-n, pt-n and pt-pt correlation coefficients with fixed impact parameter, calculated in MC model for $\mathrm{Pb}-\mathrm{Pb}$ collisions at $\sqrt{s_{N N}}=2.76 \mathrm{TeV}$ 


\section{Conclusion}

Long-range correlations are calculated in the framework of the string fusion model, taking into account finite rapidity length of the strings.

$\mathrm{N}-\mathrm{n}, \mathrm{pt}-\mathrm{n}$ and pt-pt correlation functions were calculated and it was shown that they demonstrate non-linear behaviour. The dependence of the correlation coefficients on the width of rapidity windows and the gap between them is calculated and compared with the predictions of the model of independent emitters.

The developed model enabled to describe also AA interactions without referring to the Glauber picture of nucleon-nucleon collisions. The number of soft binary collisions in this approach is proved to be less than in the Glauber approach.

Correlation coefficients for $\mathrm{Pb}-\mathrm{Pb}$ collisions are estimated in case of fixed impact parameter. $\mathrm{In} \mathrm{Pb}-\mathrm{Pb}$ collisions at LHC energy the strength of pt-pt correlation is larger compared to pt-n correlation.

\section{Acknowledgments}

The authors are grateful to M.A. Braun, G.A. Feofilov, T.A. Drozhzhova and I.G. Altsybeev for numerous useful discussions. This work was partially supported by the RFFI grant 12-02-00356-a.

\section{References}

[1] A. Capella, U.P. Sukhatme, C.I. Tan, Van J. Tran Thanh, Dual parton model, Phys. Rep., 236, 225-329, 1994

[2] H. J. Drescher, M. Hladik, S. Ostapchenko, T. Pierog, K. Werner, Parton-based Gribov-Regge theory, Phys. Rept., 350, 93-289, 2001, hep-ph/0007198

[3] M. Braun, C. Pajares, Particle production in nuclear collisions and string interactions, Physics Letters B, 287, $154-158,1992$

[4] M. Braun, C. Pajares, A Probabilistic model of interacting strings, Nucl. Phys. B, 390, 542-558, 1993

[5] M. A. Braun, C. Pajares, Implications of color-string percolation on multiplicities, correlations, and the transverse momentum, Eur. Phys. J. C, 16, 349-359, 2000

[6] V. Vechernin, R. Kolevatov, On multiplicity and transverse-momentum correlations in collisions of ultrarelativistic ions, Phys. Atom. Nucl., 70, 1797-1808, 2007

[7] V. Vechernin, R. Kolevatov, Long-range correlations between transverse momenta of charged particles produced in relativistic nucleus-nucleus collisions, Phys. Atom. Nucl., 70, 1809-1818, 2007

[8] V. Vechernin, I. Lakomov, A. Puchkov, Mean transverse momentum, multiplicity and their correlation in pp collisions in string fusion model, Vestnik SPbU, ser. 4, vyp. 3, 2010

[9] V. Kovalenko, Modelling of exclusive parton distributions and pp interaction features in the approach with the colour strings formation, Proc. "Science and Progress", pages 257-261, 2010

[10] V. Kovalenko, Modelling of exclusive parton distributions and long-range rapidity correlations for pp collisions at the LHC energy, 2012, arXiv:1211.6209 [hep-ph] 
[11] A. B. Kaidalov, O. I. Piskunova, Inclusive spectra of baryons in the quark-gluon strings model, Zeit. fur Phys. C, 30, 145-150, 1986

[12] G. H. Arakelyan, A. Capella, A. B. Kaidalov, Y. M. Shabelski, Baryon number transfer in hadronic interactions, Eur. Phys. J. C, 26, 81-90, 2002, hep-ph/0103337

[13] C. Flensburg, G. Gustafson, L. Lonnblad, Elastic and quasi-elastic pp and $\gamma^{*} p$ scattering in the Dipole Model, Eur. Phys. J. (C), 60, 233-247, 2009, arXiv: 0807.0325

[14] G. Gustafson, Multiple Interactions, Saturation, and Final States in pp Collisions and DIS, Acta Phys. Polon. B, 40, 1981-1996, 2009, comments: 15 pages. Cracow Epiphany Conference January 5-7 2009, 0905.2492

[15] C. Flensburg, G. Gustafson, Fluctuations, Saturation, and Diffractive Excitation in High Energy Collisions, ArXiv e-prints, 2010, arXiv: 1004.5502

[16] V. Vechernin, R. Kolevatov, Simple cellular model of long range multiplicity and p(t) correlations in high-energy nuclear collisions, Vestnik SPbU, ser.4, no.2, (2004) 12, hep-ph/ 0304295

[17] M. Braun, R. Kolevatov, C. Pajares, V. Vechernin, Correlations between multiplicities and average transverse momentum in the percolating color strings approach, Eur. Phys. J. C, 32, 535-546, 2004, hep-ph/0307056

[18] V. Vechernin, I. Lakomov, The dependence of the number of pomerons on the impact parameter and the long-range rapidity correlations in pp collisions, Proc. Baldin ISHEPP XXI, 2012, POS (BaIdin ISHEPP XXI) 072

[19] I. Altsybeev, G. Feofilov, M. Kompaniets, V. Kovalenko, V. Vechernin, I. Vorobyev, A. Zarochentsev, Grid technologies in SPbSU long-range correlations analysis and MC simulations for ALICE, Proc. Distributed Computing and Grid-technologies in Science and Education, Dubna, JINR, 2012

[20] P.A. Bolokhov, M.A. Braun, G.A. Feofilov, V.P. Kondratiev, V.V. Vechernin, Long-Range Forward-Backward pt and Multiplicity Correlations Studies in ALICE, ALICE Internal Note/PHY, ALICE-INT-2002-20, 1.0, 2002

[21] K. Aamodt, et al. (ALICE Collaboration), Charged-particle multiplicity measurement in proton-proton collisions at sqrt(s) $=7$ TeV with ALICE at LHC, Eur. Phys. J. C, 68, 345-354, 2010, 1004.3514 [hep-ex]

[22] N. Armesto, M. A. Braun, E. G. Ferreiro, C. Pajares, Percolation Approach to Quark-Gluon Plasma and J-psi Suppression, Phys. Rev. Lett., 77, 3736-3738, 1996

[23] V. Vechernin, R. Kolevatov, Cellular approach to long range $p(t)$ and multiplicity correlations in the string fusion model, Vestnik SPbU, ser.4, no.4, (2004) 11, hep-ph/ 0305136

[24] V. Khachatryan, et al. (CMS Collaboration), Transverse-momentum and pseudorapidity distributions of charged hadrons in pp collisions at sqrt(s) = 7 TeV, Phys. Rev. Lett., 105, 022002, 2010, 1005.3299 [hep-ex]

[25] V. Vechernin, Long-Range Rapidity Correlations in the Model with Independent Emitters, Proc. XX Baldin ISHEPP, 2010, 1012.0214 [hep-ph ]

[26] R. Kolevatov, V. Vechernin, Multiplicity and pt correlations in AA-interactions at high energies, Proc. XVIIIth QFTHEP Workshop, 2005, hep-ph/0502069

[27] G. Feofilov, Long-Range (Forward-Backward) Pt and Multiplicity Correlations in pp Collisions at 0.9 and 7 TeV, Quark Matter 2011 report,

https://indico.cern.ch/contributionDisplay.py?contribId=596\&sessionId $=55 \&$ confId $=30248$ 
[28] G. Feofilov, I. Altsybeev, V. Vechernin, S. De, T. Nayak, B. K. Srivastava, Forward-backward multiplicity correlations in pp collisions in ALICE at 0.9, 2.76 and 7 TeV, Proc. Baldin ISHEPP XXI, 2012, POS (Baldin ISHEPP XXI) 075

[29] M. L. Miller, K. Reygers, S. J. Sanders, P. Steinberg, Glauber modeling in high energy nuclear collisions, Ann. Rev. Nucl. Part. Sci., 57, 205-243, 2007, nucl-ex/0701025

[30] U. W. F. Antinori, Heavy-ion collisions - Selected topics, Proceedings of the International School of Physics Enrico Fermi, 178, 2011

[31] G. Feofilov, A. Ivanov, Number of nucleon-nucleon collisions vs. energy in modified Glauber calculations, J. Phys. CS, 5, 230, 2005

[32] K. Aamodt, et al. (ALICE Collaboration), Centrality dependence of the charged-particle multiplicity density at mid-rapidity in Pb-Pb collisions at sqrt $(s N N)=2.76 \mathrm{TeV}$, Phys. Rev. Lett., 106, 032301, 2011, 1012.1657 [nucl-ex]

[33] N. Amelin, N. Armesto, C. Pajares, D. Sousa, Monte Carlo model for nuclear collisions from SPS to LHC energies, Eur. Phys. J. C, 22, 149-163, 2001, hep-ph/0103060

[34] M. A. Braun, F. del Moral, C. Pajares, Centrality dependence distributions of the multiplicity and transverse momentum at RHIC and LHC and the percolation of strings, Nucl. Phys. A, 15, 79, 2003

[35] N. Amelin, M. Braun, C. Pajares, Multiple production in the Monte Carlo string fusion model, Phys. Lett. B, 306, 312-318, 1993

[36] V. Vechernin, On participants number fluctuations for given centrality AA-interactions in the classical Glauber approach, Proc. Baldin ISHEPP XVIII, 2007, arXiv: hep-ph / 0702141

[37] V. Vechernin, H. Nguyen, Fluctuations of the number of participants and binary collisions in AA interactions at fixed centrality in the Glauber approach, Phys. Rev. C, 84, 054909, 2011, 1102.2582 [hep-ph] 


\section{Appendix}

At fig. 14 an example of n-n correlation cloud is shown. The colour denotes the number of events with given $n_{F}$ and $n_{B} . \mathrm{N}-\mathrm{n}$ correlation function is obtained by regression of this histogram.

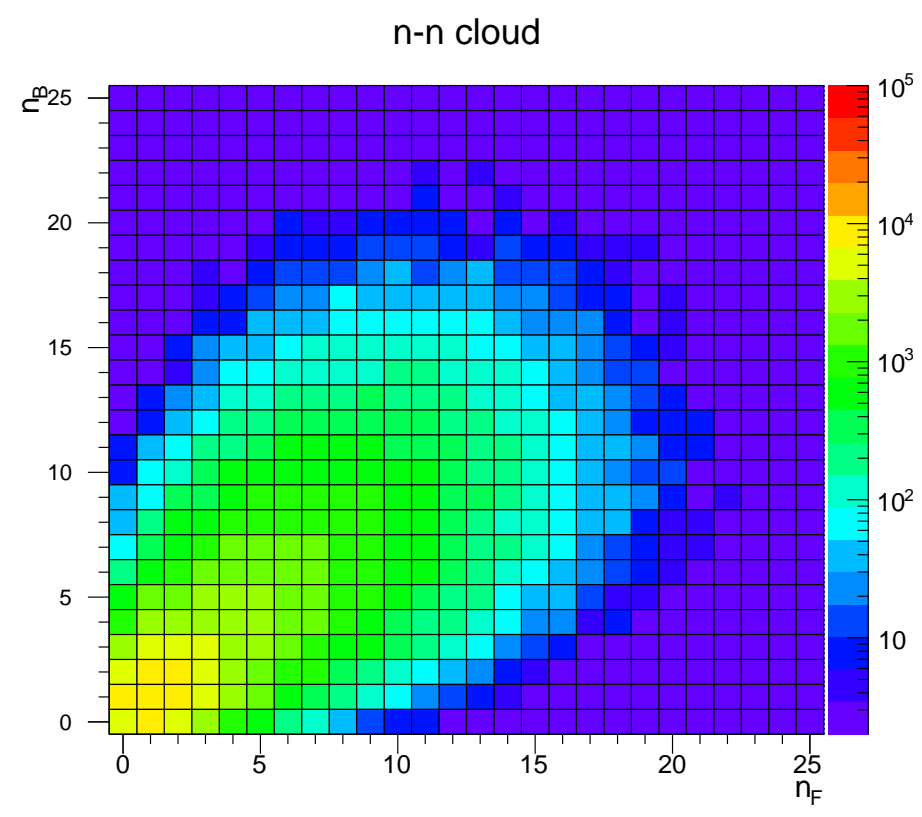

Figure 14: $2 \mathrm{~d} n-\mathrm{n}$ correlation histogram, calculated in Monte Carlo model for pp interactions at $7 \mathrm{TeV}$, rapidity windows are $(-0.8,0),(0,0.8)$. 